\title{
Lynx Mission concept status
}

\section{Jessica A. Gaskin, Ryan Allured, Simon R. Bandler, Stefano Basso, Marshall W. Bautz, et al.}

Jessica A. Gaskin, Ryan Allured, Simon R. Bandler, Stefano Basso, Marshall W. Bautz, Michael F. Baysinger, Michael P. Biskach, Tyrone M. Boswell, Peter D. Capizzo, Kai-Wing Chan, Marta M. Civitani, Lester M. Cohen, Vincenzo Cotroneo, Jacqueline M. Davis, Casey T. DeRoo, Michael J. DiPirro, Alexandra Dominguez, Leo L. Fabisinski, Abraham D. Falcone, Enectali Figueroa-Feliciano, Jay C. Garcia, Karen E. Gelmis, Ralf K. Heilmann, Randall C. Hopkins, Thomas Jackson, Kiranmayee Kilaru, Ralph P. Kraft, Tianning Liu, Ryan S. McClelland, Randy L. McEntaffer, Kevin S. McCarley, John A. Mulqueen, Feryal Özel, Giovanni Pareschi, Paul B. Reid, Raul E. Riveros, Mitchell A. Rodriguez, Justin W. Rowe, Timo T. Saha, Mark L. Schattenburg, Andrew R. Schnell, Daniel A. Schwartz, Peter M. Solly, Robert M. Suggs, Steven G. Sutherlin, Douglas A. Swartz, Susan Trolier-McKinstry, James H. Tutt, Alexey Vikhlinin, Julian Walker, Wonsik Yoon, William W. Zhang, "Lynx Mission concept status," Proc. SPIE 10397, UV, X-Ray, and Gamma-Ray Space Instrumentation for Astronomy XX, 103970S (19 September 2017); doi: 10.1117/12.2273911 


\title{
Lynx Observatory and Mission Concept Status
}

Jessica A. Gaskin ${ }^{* a}$, Ryan Allured ${ }^{\mathrm{b}}$, Simon R. Bandler ${ }^{\mathrm{c}}$, Stefano Basso ${ }^{\mathrm{d}}$, Marshall W. Bautz, Michael F. Baysinger ${ }^{\mathrm{f}}$, Michael P. Biskach ${ }^{\mathrm{g}}$, Tyrone M. Boswell ${ }^{\mathrm{a}}$, Peter D. Capizzo ${ }^{\mathrm{f}}$, Kai-Wing Chan $^{\text {h}}$, Marta M. Civitani ${ }^{\mathrm{d}}$, Lester M. Cohen ${ }^{\mathrm{b}}$, Vincenzo Cotroneo $^{\mathrm{b}}$, Jacqueline M. Davis $^{\mathrm{a}}$, Casey T. DeRoo $^{\mathrm{b}}$, Michael J. DiPirro ${ }^{\mathrm{c}}$, Alexandra Dominguez ${ }^{\mathrm{a}}$, Leo L. Fabisinski ${ }^{\mathrm{f}}$, Abraham D. Falcone ${ }^{\mathrm{i}}$, Enectali Figueroa-Feliciano ${ }^{\mathrm{j}}$, Jay C. Garcia ${ }^{\mathrm{f}}$, Karen E. Gelmis ${ }^{\mathrm{a}}$, Ralf K. Heilmann ${ }^{\mathrm{e}}$, Randall C. Hopkins $^{\mathrm{a}}$, Thomas Jackson ${ }^{\mathrm{i}}$, Kiranmayee Kilaru ${ }^{\mathrm{k}}$, Ralph P. Kraft ${ }^{\mathrm{b}}$, Tianning Liu ${ }^{\mathrm{i}}$, Ryan S.

McClelland $^{\mathrm{g}}$, Randall L. McEntaffer ${ }^{\mathrm{i}}$, Kevin S. McCarley ${ }^{\mathrm{a}}$, John A. Mulqueen ${ }^{\mathrm{a}}$, Feryal Özel ${ }^{1}$, Giovanni Pareschi ${ }^{\mathrm{d}}$, Paul B. Reid ${ }^{\mathrm{b}}$, Raul E. Riveros ${ }^{\mathrm{h}}$, Mitchell A. Rodriguez ${ }^{\mathrm{f}}$, Justin W. Rowe ${ }^{\mathrm{f}}$, Timo T. Saha ${ }^{\mathrm{c}}$, Mark L. Schattenburg ${ }^{\mathrm{e}}$, Andrew R. Schnell ${ }^{\mathrm{a}}$, Daniel A. Schwartz ${ }^{\mathrm{b}}$, Peter M. Solly ${ }^{\mathrm{g}}$, Robert M. Suggs ${ }^{\mathrm{a}}$, Steven G. Sutherlin ${ }^{\mathrm{a}}$, Douglas A. Swartz ${ }^{\mathrm{k}}$, Susan Trolier-McKinstry ${ }^{\mathrm{i}}$, James H. Tutt ${ }^{\mathrm{i}}$, Alexey Vikhlinin ${ }^{\mathrm{b}}$, Julian Walker ${ }^{\mathrm{i}}$, Wonsik Yoon ${ }^{\mathrm{m}}$, William W. Zhang ${ }^{\mathrm{c}}$

${ }^{a}$ NASA Marshall Space Flight Center, Huntsville, AL, 35812, USA

${ }^{\mathrm{b}}$ Smithsonian Astrophysical Observatory, Cambridge, MA 02138, USA

${ }^{c}$ NASA Goddard Space Flight Center, Greenbelt, MD 20771, USA

${ }^{\mathrm{d} O s s e r v a t o r i o ~ A s t r o n o m i c o ~ d i ~ B r e r a, ~ M e r a t e, ~ L C, ~ I T A ~}$

${ }^{\mathrm{e} M I T}$ Kavli Institute for Astrophysics and Space Research, Cambridge, MA 02139, USA

fJacobs ESSSA, Marshall Space Flight Center, Huntsville, AL 35812, USA

${ }^{\text {g}}$ Stinger Ghaffarian Technologies, Inc., Goddard Space Flight Center, Greenbelt, MD 20771, USA

${ }^{\text {h}}$ University of Maryland Baltimore Co., Goddard Space Flight Center, Greenbelt, MD 20771, USA

iPennsylvania State University, University Park, PA 16802, USA

${ }^{\mathrm{j} N o r t h w e s t e r n ~ U n i v e r s i t y, ~ E v a n s t o n, ~ I L, ~ 60208, ~ U S A ~}$

${ }^{k}$ Universities Space Research Association, MSFC, Huntsville, AL 35812, USA

${ }^{1}$ University of Arizona Department of Astronomy and Steward Observatory, Tucson, Arizona 85721

${ }^{m}$ Universities Space Research Association, GSFC, Greenbelt, MD 20771, USA

\begin{abstract}
Lynx is a concept under study for prioritization in the 2020 Astrophysics Decadal Survey. Providing orders of magnitude increase in sensitivity over Chandra, Lynx will examine the first black holes and their galaxies, map the large-scale structure and galactic halos, and shed new light on the environments of young stars and their planetary systems. In order to meet the Lynx science goals, the telescope consists of a high-angular resolution optical assembly complemented by an instrument suite that may include a High Definition X-ray Imager, X-ray Microcalorimeter and an X-ray Grating Spectrometer. The telescope is integrated onto the spacecraft to form a comprehensive observatory concept. Progress on the formulation of the Lynx telescope and observatory configuration is reported in this paper.
\end{abstract}

Keywords: Lynx X-ray Observatory, X-ray Surveyor, Large Mission Concept

* jessica.gaskin@nasa.gov; phone 1256 961-7818

UV, X-Ray, and Gamma-Ray Space Instrumentation for Astronomy XX, edited by Oswald H. Siegmund, Proc. of SPIE Vol. 10397, 103970S · () 2017 SPIE · CCC code: 0277-786X/17/\$18 · doi: 10.1117/12.2273911 


\section{INTRODUCTION}

Currently in its concept phase, the Lynx Observatory and mission design will accommodate an exciting science case that includes observing the first black holes and identifying the processes that will shape our view of galaxy formation and evolution. This concept, which includes the Lynx telescope (consisting of an optical assembly and science instrument suite), mission concept, and technology maturation plan, will be provided to the Astrophysics Decadal committee for review and consideration in the 2020 Survey. A NASA appointed Science and Technology Definition Team (STDT), led by two community chairs and supported by a Study Office is responsible for carrying out this Study. The Lynx Study Office is a partnership between NASA Marshall Space Flight Center (MSFC) and the Smithsonian Astrophysical Observatory (SAO). Multiple Science Working Groups (SWGs), a Communications WG (CWG), an Instrument WG (IWG), and an Optics WG (OWG) have been also been formed to support this effort. The OWG and IWG are essential to defining the Lynx telescope elements, while spacecraft design and mission concept formulation are being carried out by the MSFC Advanced Concept Office (ACO) under the guidance of the Lynx Study Office and STDT.

The Lynx science case requires an X-ray telescope that consists of a fine angular-resolution, large area optical assembly coupled to a science instrument suite that is capable of fine imaging and high-resolution spectroscopy. Multiple optical assembly studies, each using a different X-ray mirror technology, are underway, and these will enable the Lynx Team to define multiple paths towards achieving the science requirements. Similarly, the science instrument suite is undergoing multiple instrument design studies at MSFC and Goddard Space Flight Center (GSFC). The instruments under consideration (subject to change as the science case evolves) currently include of a high angular resolution megapixel imaging array, a large-format high-spectral-resolution small-pixel array, and grating arrays for high-resolution spectroscopy.

Spacecraft elements are being designed in parallel to the telescope elements, and a single team within the ACO at MSFC is carrying out the design work for both. This integrative approach allows for updates and refinements to be made in a streamlined and efficient manner.

\section{LYNX SCIENCE THEMES}

The Lynx STDT has defined two main science themes that drive the architecture for the observatory: The Dawn of Black Holes and The Invisible Drivers of Galaxy Formation and Evolution. These themes highlight the Lynx unique observations that will provide new insight into the formation and evolution of the Universe.

\subsection{The Dawn of Black Holes}

Lynx will observe the birth of the first seed black holes and provide a census of the massive black hole population in the local and distant universe, follow their growth and assembly across cosmic time, and measure the impact of their energy input on all scales. The very existence of billion-solar-mass black holes at a time when the Universe was only a few percent of its current age shows that either they began as very massive "seeds" or they underwent a very early phase of sustained and rapid growth. Directly detecting these seed black holes near the moment of their creation will provide the cleanest test of the physics of seed formation. This implies that we need to push as deep as possible to detect very faint sources at very high redshifts. Lynx will be the ultimate machine for black hole studies.

\subsection{The Invisible Drivers of Galaxy Formation}

The assembly, growth, and the state of visible matter in the cosmic structures is largely driven by violent processes that heats the gas in the circumgalactic and intergalactic media. The exquisite spectral and angular resolution of Lynx will make it a unique instrument for mapping the hot gas around galaxies and in the Cosmic Web. The hot circumgalactic medium is both the reservoir for galaxy-building material and the repository for metals and energy liberated by energetic processes in the cores of galaxies - from stellar winds to supermassive black hole outflows. Lynx will reveal the complex cycling of baryons from stars into this hot gas and back, and provide our first detailed view of the elusive warm-hot intergalactic medium that we expect to fill the space beyond galaxies. Tracing the energy input and measuring the thermodynamic state of the circumgalactic gas is best done in the X-rays with Lynx, where the dynamics of the hot ambient phase and spectroscopic signatures of multi-temperature gases and abundances can be traced with highresolution X-ray spectroscopy and high angular resolution imaging. 


\section{HIGH-RESOLUTION OPTICS ASSEMBLY}

The Lynx science themes drive the requirements for the optical assembly (Table 1). The Lynx telescope will rely on grazing-incidence optics using two reflections to bring X-rays in the $\sim 0.1 \mathrm{keV}$ to $\sim 10 \mathrm{keV}$ energy range to focus. A large effective collecting area is achieved by nesting very large numbers of co-aligned, co-axial mirror pairs in order to optimize the available aperture. Currently, the Lynx optical assembly is envisioned to be approximately $3 \mathrm{~m}$ diameter and will have a $10 \mathrm{~m}$ focal length.

Table 1. Lynx Optical assembly requirements.

\begin{tabular}{ll}
\hline Element & Requirement \\
Energy Range & $\sim 0.1 \mathrm{keV}$ to $\sim 10 \mathrm{keV}$ \\
Angular resolution (on-axis) & $0.5 \operatorname{arcsec} \mathrm{HPD}$ (or better) \\
Effective area & $2 \mathrm{~m}^{2} @ 1 \mathrm{keV}$ \\
Off-axis PSF (grasp), $\mathrm{A} *(\mathrm{FOV}$ for HPD $<1$ arcsec) & $\sim 600 \mathrm{~m}^{2} \operatorname{arcmin}^{2}$ \\
\hline
\end{tabular}

Large effective area is achieved by requiring that Lynx optics are $\sim 0.5 \mathrm{~mm}$ up to a few $\mathrm{mm}$ thick and preferably made of a lightweight material (e.g., glass, silicon, or light-weighted metal alloy substrate material). These optics allow for a high degree of nesting and acceptably low mass for launch considerations. Currently, NASA funds several R\&D programs to develop such optics for future X-ray missions. Many of these efforts are summarized in (O'Dell et al., 2016) ${ }^{1}$.

The Lynx team is studying multiple X-ray optics technologies that will be able to meet Lynx requirements and are focusing on full shell, adjustable segmented and meta-shell silicon optics technologies in particular. A detailed optical design and technology maturation plan is being developed for each. These plans include, but are not limited to, defining a process for inexpensive and rapid fabrication of large numbers of precisely figured mirrors, a maturation and fabrication schedule, a plan for quality control, and logistics constraints for manufacturing, calibration and testing.

\subsection{Full Shell Optics}

Direct fabrication of full shell high-angular resolution X-ray optics was first realized for the Einstein telescope and then adopted for the Chandra X-ray Observatory. Using this process, Chandra was able to obtain the exquisite angular resolution $(0.5 \text { arcsecond } \mathrm{HEW})^{2}$ that has facilitated numerous scientific discoveries. Lynx, however, because of its larger effective area requirement, requires thinner optics than those used on Chandra, posing some challenges. These include stress induced from grinding, polishing and coating the substrate, as well as mounting and structural challenges ${ }^{1}$.

Two groups are co-leading the Lynx full-shell study, and they are located at NASA MSFC and the Astronomical Observatory of Brera. Both groups have on-going funded efforts to develop few-mm thick, high-angular resolution, direct fabricated optics. Because the optics developed by Brera are more mature in their development, they have been selected for use in this study (Figure 1).

Brera/Instituto Nazionale Di Astrofisica (Brera/INAF) has successfully fabricated a 2-mm-thick full-shell mirror with a monolithic structure ${ }^{3}$ with an angular resolution $<20$ arcseconds almost constant across a field of view of $1 \mathrm{deg}$ diameter. The technology development is focused on using fused silica for their substrate material, and their fabrication technique capitalizes on the Chandra approach ${ }^{4}$. Fused-silica shells are first ground to a double-cone profile with a shell thickness of a few millimeters. Then a fine grinding process is pursued in order to impart the correct azimuthal and longitudinal profiles. After this, bonnet polishing and classical superpolishing operations, applied directly on the high precision lathe used for the fine grinding or using a computer-controlled Zeeko IRP600X CNC polishing machine, obtain the finishing of the surfaces. The "bonnet" polishing technique is based on a high velocity precessing rubber hemispherical diaphragm ${ }^{5}$. The computer-controlled Zeeko polishing machine and the shape of bonnet polishing lap provides precise control of the polishing parameters, such as the wear function rate, shape, and footprint, potentially leading to a faster convergence compared to traditional polishing machines. During these operations, the shell is supported with a series of mechanical flexures around the circumference of the shell at each end. For the Brera Observatory approach, it is expected that the use of the highly deterministic polishing process will eliminate all low- 
spatial-frequency errors and lower the mid-frequency errors to the point that ion figuring can correct the residual errors. Near-term efforts include the development of an optomechanical design of the Lynx telescope based on thin close glass shells ${ }^{6}$.

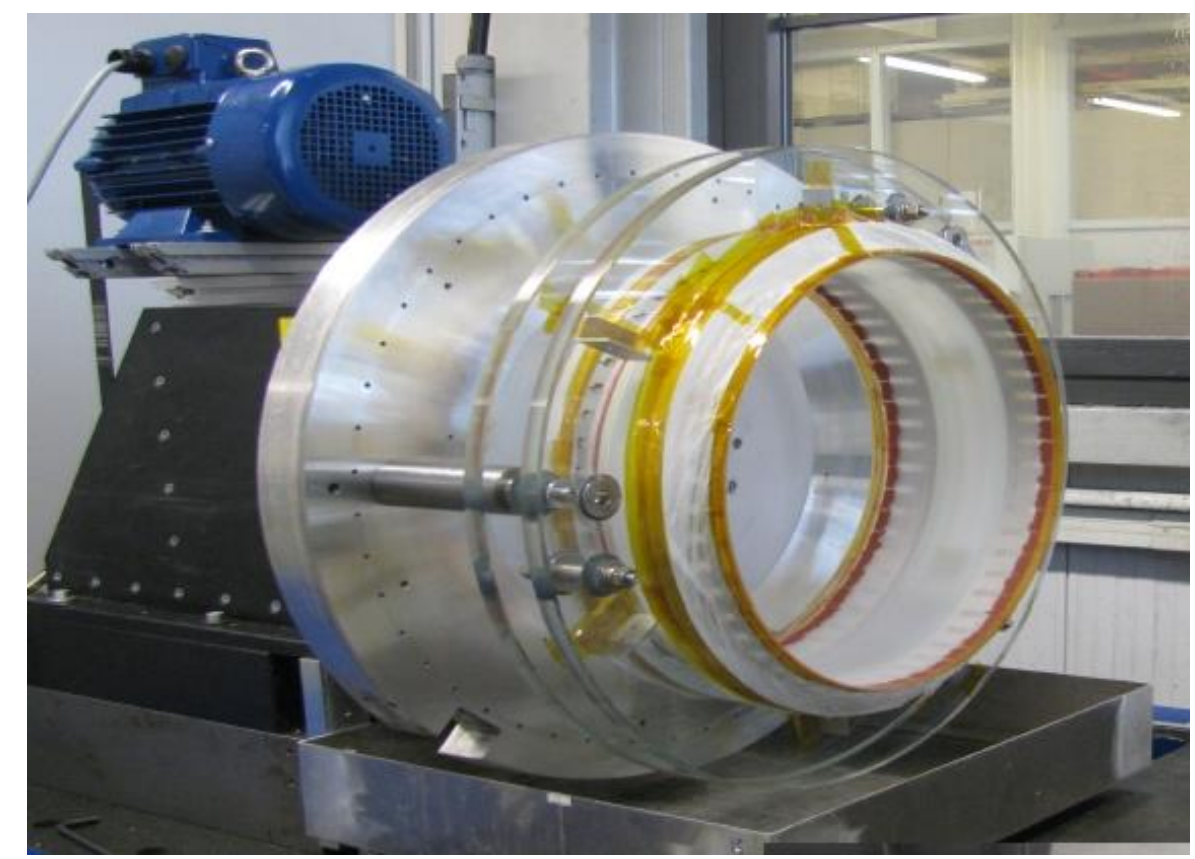

Figure 1. Photograph of a 2-mm thick, 486.5-mm diameter full-shell fused silica optic mounted on a fixture developed by Brera/INAF [credit: Brera/INAF].

\subsection{Adjustable Optics}

The Smithsonian Astronomical Observatory (SAO) is leading the Lynx adjustable segmented optics team. Adjustable segmented optics employ thin film piezoelectric adjusters to correct mirror figure for fabrication errors, mounting induced distortions, and potential on-orbit changes due to thermal environment. The process considered for Lynx requires that thin $(\sim 0.5 \mathrm{~mm})$, slumped-glass segments be treated with electro-active coatings (Figure 2). There are multiple funded, on-going efforts, including work at SAO and Northwestern University (employing magneto-restrictive films) reporting good progress ${ }^{7,8}$.

SAO and PSU have recently successfully tested a cylindrical test mirror coated with 112 piezo-actuators, demonstrating feasibility of this process on curved X-ray mirrors. Adjuster voltages were optimized via computer modeling and then applied to minimize the mirror root-mean-squared (RMS) axial slope error in the mirror. The test mirror was used to demonstrate a measured figure change correcting an estimated 6.7 arcseconds HPD mirror figure (single reflecton @ 1 $\mathrm{keV}$ ) to 1.2 arcseconds HPD, close to the predicted correction performance of 1.3 arcseconds HPD. The predicted correction was based on an optimized set of actuator voltages obtained using measured influence functions, which show good agreement between the influence functions of a modeled mirror given metrology noise ${ }^{9}$. Predicted performance without the relatively large amount of metrology noise was 0.3 arcsecond HPD, suggesting that noise filtering techniques for the influence functions will result in a substantial improvement in performance.

On-going efforts at SAO include improving the quality of the underlying glass substrate, minimizing stress in the applied electro-active coatings, mounting of these optics into a subassembly and assembly suitable for Lynx and application of integrated thin-film transistor arrays for row-column addressing of the piezoelectric pixels, which will substantially reduce the number of electrical connections to the mirror. 

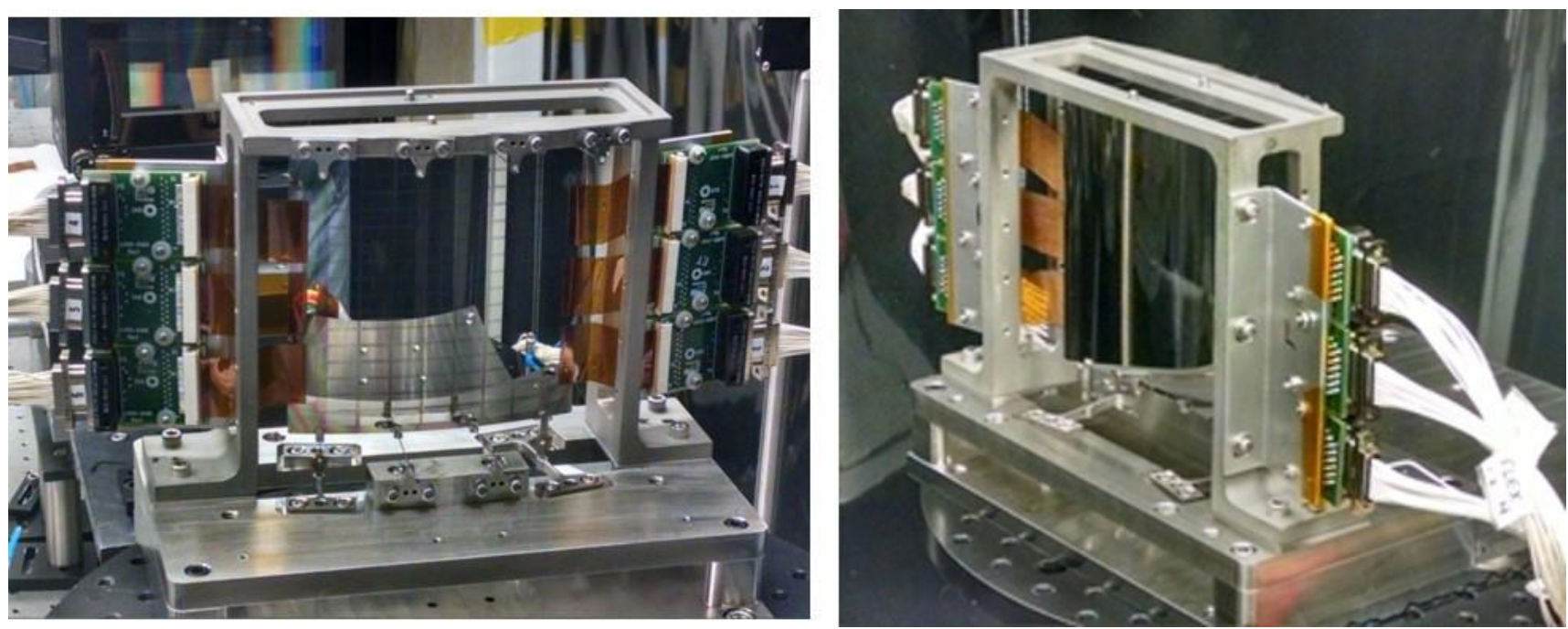

Figure 2. SAO adjustable optics. (Left) This image shows the back of the optic. The electrodes are visible, and are in a pixel-like pattern. (Right) This image shows the X-ray reflecting side of the optic [credit: SAO].

\subsection{Mono-Crystalline Si Meta-Shell Optics}

NASA GSFC is leading the silicon meta-shell segmented approach ${ }^{10}$ for Lynx. This approach has two distinct features. First, it allows for the fabrication of mirror segments that meet performance requirements by combining the direct fabrication technique with the stress-free nature of mono-crystalline silicon to make high-resolution, lightweight and thin $(\leq 1 \mathrm{~mm}) \mathrm{X}$-ray optics. Mono-crystalline silicon is free of stress and has good thermo-mechanical properties (e.g., high thermal conductivity, low CTE, and higher elastic modulus than glass does). As of August 2017, GSFC has developed a fabrication process, similar to the semiconductor industry's wafer fabrication process ${ }^{11}$ that is capable of making lightweight silicon mirror segments meeting or close to meeting Lynx's performance requirements: $\sim 0.5$ arcsecond HPD (two reflections). Second, this approach aligns and bonds each mirror segment at four locations to form a meta-shell. Each mirror segment is kinematically supported during the alignment process and minimally constrained when bonded with epoxies ${ }^{12}$ (Figure 3). Structural analyses and vibration tests have shown that this way of bonding mirror segments can meet spaceflight environmental requirements.
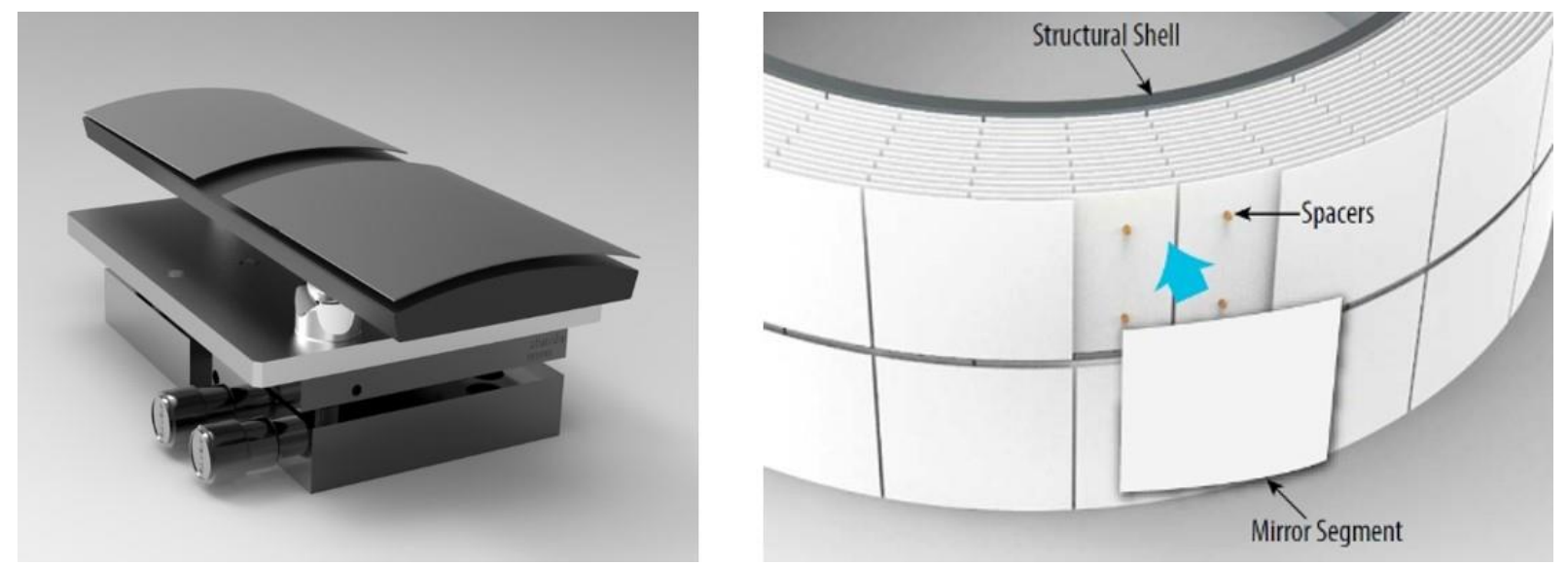

Figure 3. (Left) A single pair of mirrors that have been aligned, bonded, and tested in an X-ray beam, demonstrating the validity of the four-point alignment and bonding concept. (Right) An artist's rendition of a meta-shell consisting of many mirror segments aligned and bonded using the same method [credit: NASA GSFC]. 
Recent X-ray measurements were made on a single pair of uncoated, 1-mm thick mono-crystalline silicon mirrors on a technology development mount, obtaining X-ray images better than 5 arcsecond $\mathrm{HPD}^{12}$, demonstrating that this method has the potential of meeting imaging performance requirements as well. Work in the near-term will focus on improving both the mirror fabrication process and the mirror alignment and bonding process, making mirror modules with evermore mirror segments, X-ray testing as well as environmentally testing these modules.

\section{LYNX SCIENCE INSTRUMENTS}

The science instrument suite for Lynx currently consists of the HDXI (High Definition X-ray Imager), LXM (Lynx X-ray Microcalorimeter) and XGS (X-ray Grating Spectrometer). These instruments are being designed to meet the Lynx science requirements and will complement the fine angular resolution and large FOV of the optical assembly. As the Lynx science case evolves, these requirements and instrument designs will be refined.

These instruments have each undergone, or are in the process of undergoing, a detailed instrument design study. As each instrument is designed, it is integrated onto the Integrated Science Instrument Module (ISIM). In the case of the XGS, the detector array for the XGS is integrated onto a fixed platform on the ISIM, while the gratings are integrated onto the optical bench. Actuation of the XGS gratings allow them to be inserted into the optical path as required.

\subsection{High Definition X-ray Imager (HDXI)}

HDXI is a wide field ( $\sim 22 \operatorname{arcmin})$, high-resolution ( $\$ 0.33 \operatorname{arcsec} \approx 16 \mu \mathrm{m}$ pixels) X-ray photon counting imager. In its current form, HDXI will feature an array of silicon sensors and will provide imaging spectroscopy with good spectral resolution. Lynx-HDXI also requires good detection efficiency in the energy range $0.2 \mathrm{keV}<\mathrm{E}<10 \mathrm{keV}$ along with potentially high frame rates in excess of $10 \mathrm{~Hz}$ (possibly up to $100 \mathrm{~Hz}$ or more). In a small sub-field, time resolution of approximately $1 \mathrm{~ms}$ is also likely to be required. Three active pixel sensor (APS) detector types have the capacity to meet Lynx requirements, and are being considered: Digital Charge Coupled Devices (CCDs) [Massachusetts Institute of Technology-Lincoln Laboratory $]^{13}$, Hybrid Complementary Metal Oxide Semiconductor (CMOS) [Teledyne/Pennsylvania State University] $]^{14}$, and Monolithic CMOS [Sarnoff/SAO/Max-Planck Institut für Extraterrestrische Physik $]^{15,16}$. These detectors all have active research efforts and have been described extensively in the literature.

HDXI recently completed its first Instrument Design Study (IDS) within the ACO at MSFC. Study inputs (e.g., mass, power, volume, etc...) were selected to accommodate all three detector types. This study included a comprehensive design of the detector assembly layout, avionics (data processing strategy, wiring diagram cabling and packaging), power, thermal, and structures (including filter wheel).

The preliminary concept shows an array of 21 APSs, each with its own Application-Specific Integrated Circuit (ASIC) located on the Front-End Motherboard (FEMB) (Figure 4). This assembly is located inside a vacuum enclosure with a filter assembly attached to a front window. The FEMB is connected to the Detector Electronics Unit (DEU) that performs event-processing, controls the detectors, and generates the voltages needed for the system. The DEU also includes a Back End Processor (BEP) board that packages the data and sends it to the spacecraft bus for storage and transmission to the ground. The DEU has a full set of redundant boards to mitigate failure of any one function.

Thermal control of the Detector Assembly includes a heat sink for the detector array and another heat sink for the FEMB. Trim heaters are mounted to the detector heat sink directly under the sensors. Thermocouples are used to monitor the temperature on the APS array. Together, the trim heaters and thermocouples enable precision thermal control of the sensors. Thermal control also includes six temperature sensors on the FEMB and heat pipes that pass through the enclosure walls to radiators located on the ISIM. 


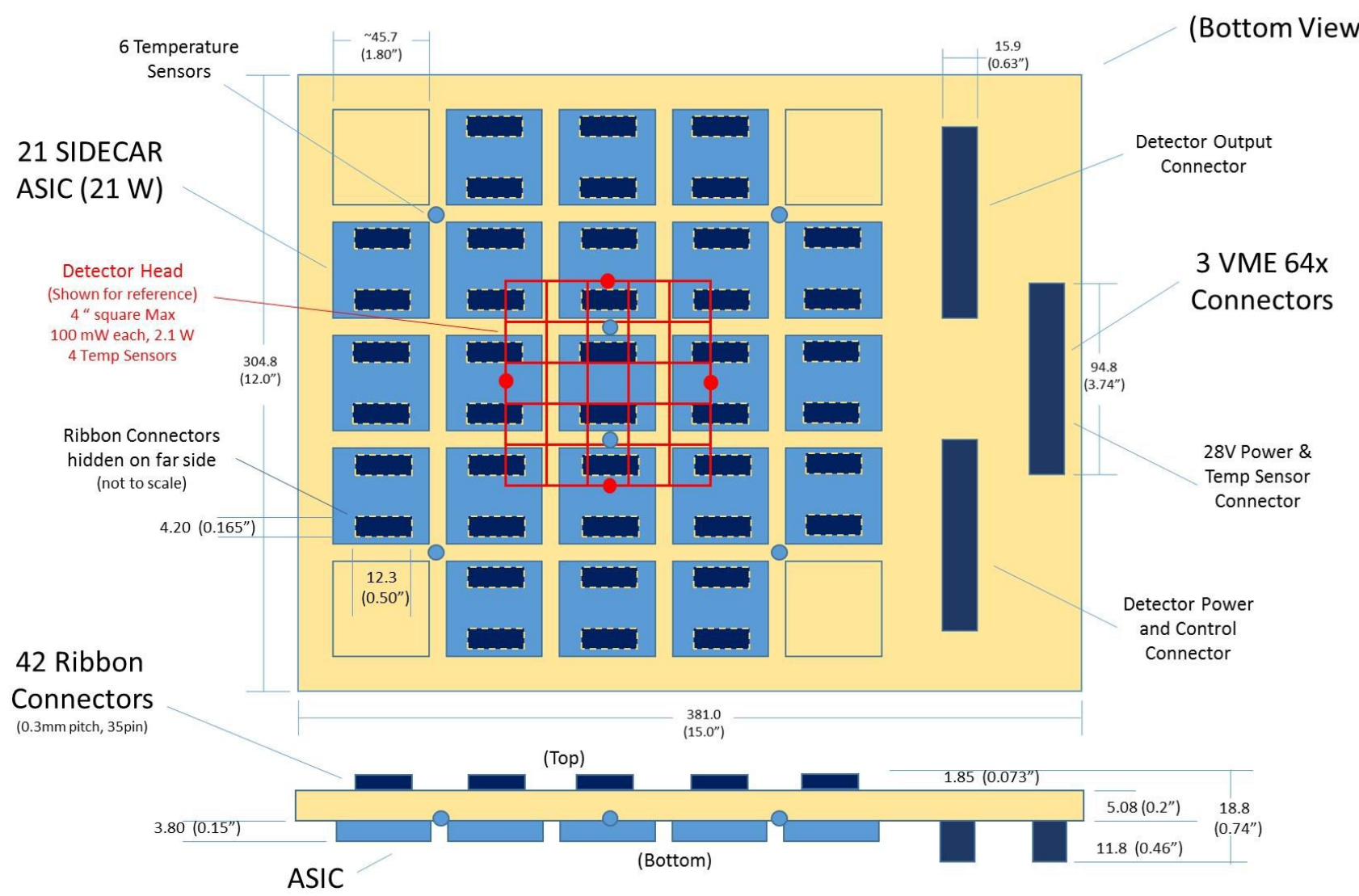

Figure 4. Preliminary Front-End Motherboard (FEMB) layout for HDXI. This concept employs an array of 21 Active Pixel Sensors coupled to their respective ASICs [credit: NASA MSFC].

A second iteration IDS at MSFC or with the Instrument Design Lab (IDL) at GSFC is being planned for further refinement.

\subsection{Lynx X-ray Microcalorimeter (LXM)}

The LXM is a cryogenic instrument with a focal plane temperature of $50-100 \mathrm{mK}$ that provides extremely high spectral resolution (< 4 eV FWHM, $0.2-10 \mathrm{keV})$, non-dispersive spectroscopy with $\sim 1$ arcsecond or better imaging capability. Lynx requires a large area sensor that will have $\sim 10^{5}$ pixels, requiring multiplexing signals from individual pixels on the focal plane to external electronics. LXM will have more capability than any other X-ray microcalorimeter that has flown in space or will fly in the near future ${ }^{17}$. However, Lessons Learned and technology developments from these predecessor missions will be invaluable to the development and operation of the LXM.

LXM completed an in-depth design study in the IDL in mid- 2017. Like the IDS for HDXI, the LXM IDL included the design of all major detector elements as well as a first estimate of the cost. Since this IDL, the LXM study leads have continued to refine the concept, the result of which is shown in Figure 5. A second IDL at GSFC or IDS at MSFC is being planned for further refinement. 

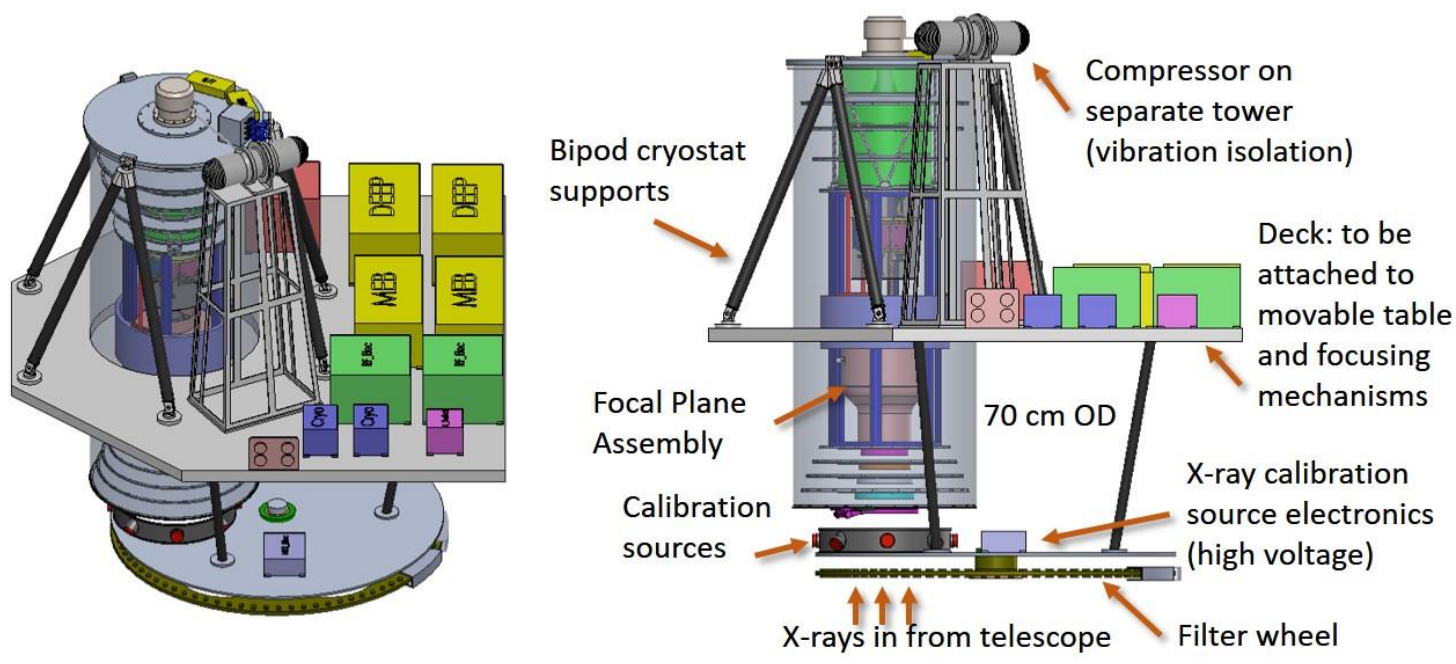

Figure 5. Rendering of the LXM with major components labeled. A more compact filter wheel (not shown here) has been designed to better accommodate integration onto the ISIM with the other science instruments [credit: NASA GSFC].

\subsection{X-ray Grating Spectrometer (XGS)}

$\mathrm{XGS}$ is an objective grating X-ray spectrometer that will provide spectral resolving power of order $\mathrm{R} \sim 5000$ or more in the $0.2 \mathrm{keV} \lesssim \mathrm{E} \lesssim 2 \mathrm{keV}$ band. Assemblies of blazed gratings will be installed on the optical bench with actuated hinges so that they can be moved into and out of the optical path of the optical assembly. The total required geometrical area of the grating assemblies of order $>1 \mathrm{~m}^{2}$ will be achieved by assembling and aligning a large number of relatively small precision grating elements in a hierarchical mounting structure. The spectrum of a target celestial source will be focused onto a sensor array mounted to a fixed location on the ISIM. This readout detector array must be able to count individual $\mathrm{X}$-ray photons and determine their energies well enough to separate overlapping spectral orders diffracted by the grating. The same technology used for HDXI will be used for this readout

Two different grating technologies, both with active research programs, are being considered for use on Lynx: Off-Plane Gratings (OPGs) (PSU) ${ }^{18,19}$ and Critical Angle Transmission (CAT) Gratings (MIT) ${ }^{20,21}$. The MSFC ACO is studying both of these technologies as each has the capacity to meet Lynx requirements. As with HDXI and LXM, a second iteration IDS or IDL will be carried out at either MSFC or GSFC, respectively next year.

\section{OBSERVATORY DESIGN STATUS}

In parallel to the instrument design studies, the MSFC ACO, working with the Lynx Study Office and STDT members, has been engaged in defining the Mission parameters (launch vehicle, orbit, etc...) and integrated Lynx Observatory (telescope + spacecraft) concept. The spacecraft design encompasses all major subsystems including propulsion, thermal control, avionics, guidance, navigation and control, electrical power, and structures and mechanisms. Mass and power studies are included, and the design is undergoing refinement through a series of planned mission design cycles.

This paper focuses mainly on the preliminary telescope mechanical configuration and the systems engineering approach used for the concept design. Specific details on the spacecraft design and mission design elements will be reported in a future publication.

\subsection{Lynx Observatory Configuration Status}

The Lynx telescope has a 10-m focal length and consists of the optical assembly mounted to the optical bench and the ISIM. The current configuration, shown in Figure 6, will to evolve, as the science case is refined. The optical assembly 
includes the multiple mirror segments or full shell mirrors and their supporting structure, as well as all of the thermal control elements (including pre- and post- thermal collimators). The optical bench is critical for maintaining alignment between the optical assembly and the focal plane instruments, and provides structure for mounting the XGS gratings, inner contamination door, and a magnetic broom to "sweep" charged particles heading towards the detectors (Figure 7).

The ISIM houses the HDXI, LXM and XGS detector assembly, and provides structure for mounting radiators. The ISIM has a moveable platform that will allow for the HDXI and LXM to translate into and out of the focus of the optics (perpendicular to the optical axis), and will permit focus adjustment (parallel to the optical axis). The ISIM also has a second fixed platform to accommodate the XGS detector assembly, electronics and associated radiators. The XGS detector assembly will have its own focus mechanism independent from that of the ISIM. Since the Lynx Team is considering both the CAT and OPG grating technologies, configurations for each are being designed.

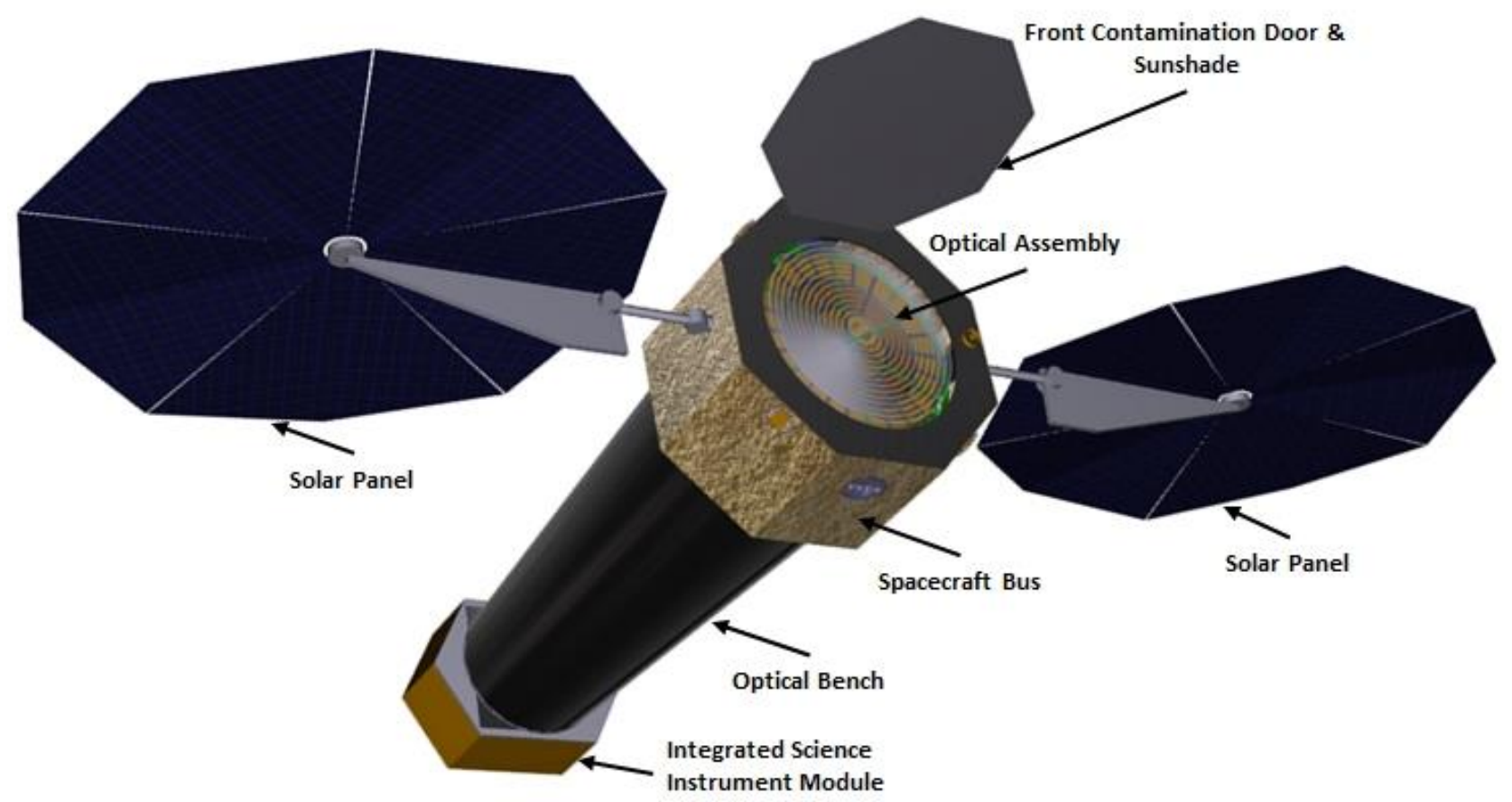

Figure 6. Current configuration for the Lynx Observatory. The Lynx telescope consists of the optical assembly connected to the Integrated Science Instrument Module (ISIM) via an optical bench. The optical assembly includes thermal control, pre- and postcollimators, while the ISIM houses the HDXI, LXM and detector for the XGS. The gratings assembly is mounted to the optical bench close to the optical assembly and inner contamination door. The spacecraft front contamination door/sunshade, spacecraft bus and solar panels is also shown [credit: NASA MSFC].

The spacecraft bus provides all of the support elements for the telescope, including propulsion, communication, navigation, command and data handling, thermal control, and power. The MSFC ACO has completed preliminary, detailed studies for these systems (including relevant trades), and has completed an extensive trade study on the Lynx orbit.

This trade study included an evaluation of potential launch vehicles, $\Delta \mathrm{V}$ for achieving orbit, thermal environment, total time spent in eclipse, communications, meteoroid environment, radiation environment, serviceability, disposal maneuver, station keeping, and disturbance environment. Orbits considered were Sun-Earth L2, Lunar Resonance 2:1, Lunar Distant Retrograde, Drift-Away, and a Chandra-Type orbit. Of these orbits, the Sun-Earth L2 and Lunar Resonance 2:1 orbits best meet Lynx requirements. 


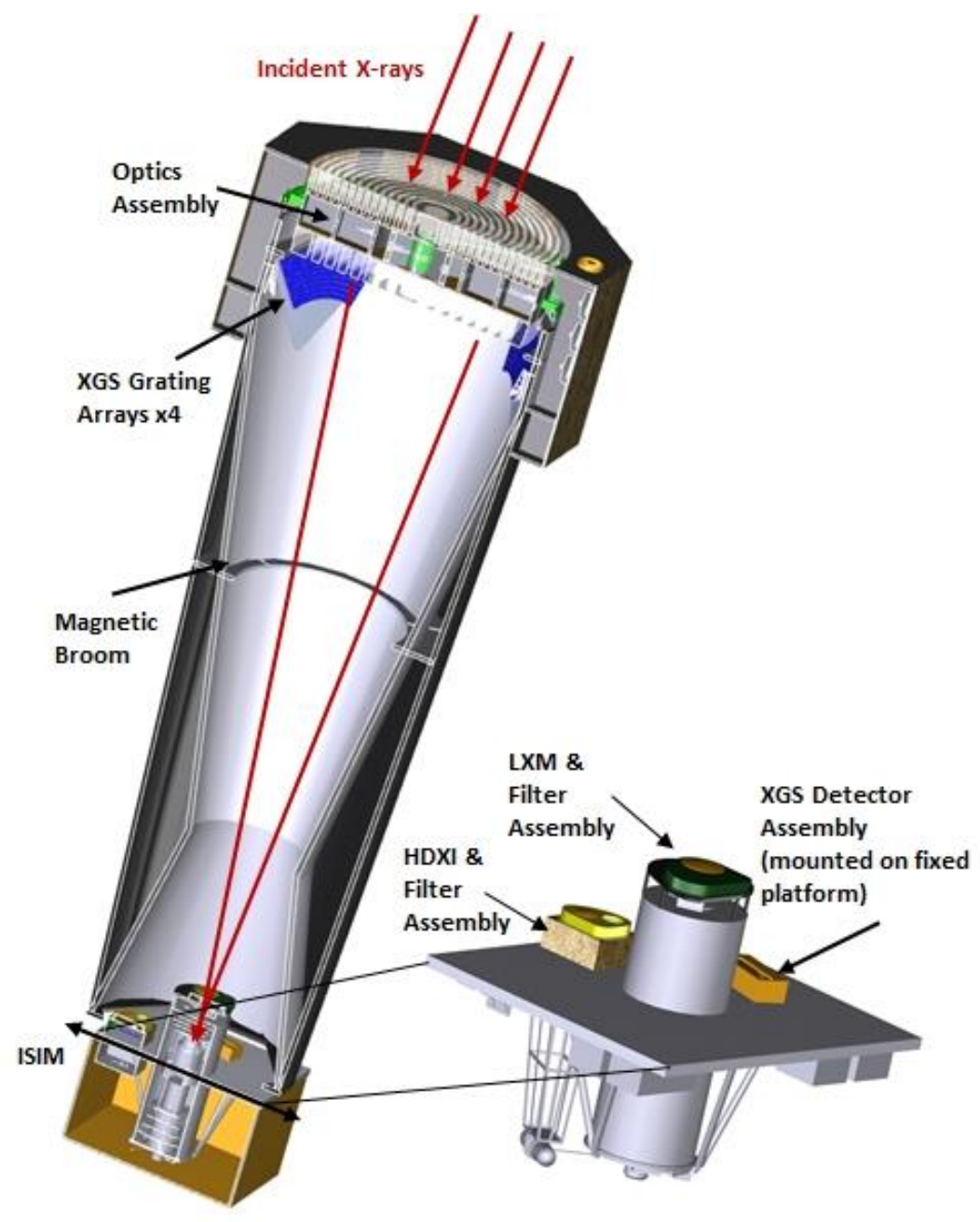

Figure 7. Cross section of the Lynx Observatory showing the optical assembly, XGS grating arrays, magnetic broom, and ISIM with the layout of the science instruments. Currently, only one instrument platform is shown for instrument layout purposes. The designs allows for the ISIM to have two platforms, one that has the ability to translate the HDXI and LXM into and out of focus and another that is fixed and located the XGS detector assembly. The inner and outer contamination doors are not shown in this view [credit: NASA MSFC].

\subsection{Model Based Systems Engineering Approach}

The overall systems engineering approach for the Lynx concept study is to utilize systems engineering expertise at the mission, optics and instrument design levels to ensure an integrated systems-level approach to balance science requirements and complexity across systems. This approach has led to the development of an integrated system architecture meeting science requirements, while maintaining a low risk posture. The Lynx systems engineering team is led by a Lead Systems Engineer (LSE) whose role is to implement trade studies and integrate the overall mission architecture design through successive mission analysis cycles. Working with the LSE are the Instrument Systems Engineer (ISE) and the Optics Systems Engineer (OSE), whose roles are to work with the LSE and Instrument and Optics teams to integrate the instrument and optics system designs into the overall architecture.

To enable the project level systems engineering approach, the Lynx SE team is utilizing Model Based Systems Engineering (MBSE) using the SysML platform. The SysML models are being developed in via a successful partnership with the University of Alabama at Huntsville's (UAH) school of Industrial and Systems Engineering. The modeling strategy is to focus on content typically documented in the Concept of Operations (ConOps). 
The development of the ConOps during this early stage of the mission will ensure that Lynx meets stakeholder expectations, that observatory elements relevant to operations are well understood, and that the functional requirements that flow from the Lynx Science case are maintained. The use of SysML modeling ensures consistency throughout the system design and provides a platform for communication and consensus on various aspects of the system across the observatory.

\section{DISCUSSION}

The Lynx Observatory and Mission design work requires support from a large team of individuals from multiple institutions (STDT members, Study Office, Instrument Working Group, Optics Working Group, among others) to realize. The majority of the work is being done within the ACO at NASA MSFC, and leverages the many years of spacecraft and mission development experience of the team facilitating the study. The ACO will continue to work with the Lynx Team through the duration of the Study to refine this design.

The Lynx science instruments preliminary in-depth studies have either been completed or are nearing completion. As the Lynx STDT refines the Science Case, additional design studies will be needed to ensure that science requirements are maintained. This is also true of the optical assembly, which will also undergo an integrated design study in the mid2018. As the science instruments and optical assembly designs are refined, the spacecraft will need to be refined to accommodate them.

\section{SUMMARY}

Lynx will be the first NASA Flagship X-ray Astronomy Mission to fly since Chandra, and will make unique measurements of the first black holes and their host galaxies, trace out and characterize the large-scale structure and galactic halos, and greatly contribute to the understanding of young stars and the formation of planetary systems, including our own solar system. The Lynx team is dedicated to ensuring that the Lynx Observatory and Mission design will provide the Astronomy Community with a new tool that will answer some of the most challenging questions pertaining to the formation and evolution of our Universe.

\section{ACKNOWLEDGMENTS}

We would like to acknowledge the many members of the Lynx Team, particularly those members who have volunteered their time to support the development of the Lynx science case, X-ray optics and science instrument studies. We would also like to thank NASA Headquarters for their support, as well as MSFC, SAO and GSFC for their Institutional contributions to this Study. 


\section{REFERENCES}

[1] O’Dell, S.L., Allured, R., Ames, A.O., Biskach, M.P., Broadway, D.M., Bruni, R.J., Burrows, D.N., Cao, J., Chalifoux, B.D., Chan, K.-W., Chung, Y.-W., Cotroneo, V., Elsner, R.F., Gaskin, J.A., Gubarev, M.V., Heilmann, R.K., Hertz, E., Jackson, T.N., Kilaru, K., Kolodziejczak, J.J., McClelland, R.S., Ramsey, B.D., Reid, P.B., Riveros, R.E., Roche, J.M., Romaine, S.E., Saha, T.T., Schattenburg, M.L., Schwartz, D.A., Schwartz, E.D., Solly, P.M., Trolier-McKinstry, S., Ulmer, M.P., Vikhlinin, A., Wallace, M.L., Wang, X., Windt, D., Yao, Y., Ye, S., Zhang, W.W., Zuo, H., "Toward large-area sub-arcsecond x-ray telescopes II," Proc. SPIE 9965, 996507 (2016).

[2] Van Speybroeck, L.P.; Jerius, D., Edgar, R. J., Gaetz, T. J., Zhao, P.; Reid, P. B, "Performance expectation versus reality," Proc. SPIE 3113, p. 89-104 (1997).

[3] Civitani, M.M., Citterio, O., Campana, S., Conconi, P., Mattaini, E., Pareschi, G., Tagliaferri, G., Parodi, G., Burwitz, V., Hartner, G.D., Arnold, J., Schuler, S., Combrinck, H., Freeman, R., Morton, R., Simpson, P., Walker, D., "Thin glass shell oriented to wide field x-ray telescope," Proc. SPIE 8443, Space Telescopes and Instrumentation 2012: Ultraviolet to Gamma Ray, 84430Q (27 September 2012)

[4] Civitani, M.M., Basso, S., Citterio, O., Hołyszko, J., Ghigo, M., Pareschi, G., Parodi, G., Toso, G. Vecchi, G., "Thin fused silica shells for high-resolution and large collecting area x-ray telescopes (like Lynx/XRS)," These Proc. 10399, Paper n. 31 (2017).

[5] Walker, D.D., Beaucamp, A.T.H., Doubrovski, V., Dunn, C., Evans, R., Freeman, R., Kelchner, J., McCavana, G., Morton, R., Riley, D., Simms, J., Yu, G., Wei, X., "Automated optical fabrication: first results from the new Precessions 1.2m CNC polishing machine", Proc. SPIE 6273, Optomechanical Technologies for Astronomy, 627309 (27 July 2006).

[6] Basso, S., Civitani, M. M., Pareschi, G. and Parodi G., "An opto-mechanical design study for a high-throughput and high-angular resolution x-ray mission (Lynx/X-Ray Surveyor like),” These Proc. 10399, Paper n. 30 (2017).

[7] Ulmer, M., Coppejans, R., Buchholz, D.B., Cao, J., Wang, W., Mercado, A.M., Qian, J., Assoufid, L., O’Donnell, A.E., Condron, K.S., Harpt, B.E., "Controlling the shapes of coated silicon substrates via magnetic fields, a progress report," This Proc., Paper 10399-59 (2017).

[8] Walker, J., Tianning, L., Tendulkar, M., Burrows, D., DeRoo, C., Allured, R., Hertz, E., Cotroneo, V., Reid, P.B., Schwartz, E.D., Jackson, T.N., Trolier-McKinstry, S., "Design and fabrication of adjustable x-ray optics using piezoelectric thin films," This Proc., paper 10399-56 (2017).

[9] DeRoo, C.T., Allured, R., Cotroneo, V., Hertz, E., Marquez, V., Reid, P.B., Schwartz, E.D., Vikhlinin, A., TrolierMcKinstry, S., Walker, J., Jackson, T.N., Liu, T., Tendulkar, M., "Deterministic figure correction of piezoelectrically adjustable slumped glass optics," This Proc., Paper 10399-58 (2017).

[10] Zhang, W.W., Allgood, K.D., Biskach, M.P., Chang, K.-W., Hlinka, M., Kearney, J.D., Mazzarella, J.R., McClelland, R.S., Numata, A., Olsen, L.G., Riveros, R.E., Saha, T.T., Solly, P.M., "Monocrystalline Silicon and the Meta-shell Approach to Building X-ray Astronomical Optics," These Proc., paper 10399-27 (2017).

[11] Riveros, R.E., Biskach, M.P., Allgood, K.D., Kearney, J.D., Hlinka, M., Zhang, W.W., "Progress on the fabrication of lightweight single crystal silicon X-ray mirrors," These Proc., paper 10399-28 (2017).

[12] Chan, K.-W., Mazzarella, J.R., Saha, T.T., Zhang, W.W., McClelland, R.S., Biskach, M.P., Riveros, R.E., Allgood, K.D., Kearney, J.D., Sharpe, M.V., Hlinka, M., Numata, A.,"Kinematic Alignment and Bonding of Silicon Mirrors for High-Resolution Astronomical X-ray Optics," These Proc., paper 10399-29 (2017).

[13] Suntharalingam, V., Burke, B., Cooper, M., Craig, D., Lambert, R., Newcomb, K., Rathman, D., Young, D., Bautz, M.W., Foster, R.F., LaMarr, B., Malonis, D., Progozhin, G.Y., "Large area digital CCDs for scientific, scanning, and staring applications," Submitted for presentation at the 2017 International Devices Meeting of the IEEE (2017).

[14] Hull, S.V., Falcone, A.D., Burrows, D.N., Wages, M., Chattopadhyay, McQuaide, M., Bray, E., Kern, M., "Recent x-ray hybrid CMOS detector developments and measurements," These Proc., paper 10397-3 (2017).

[15] Kenter, A., Kraft, R., Gauron, T., Amato, S., "Advancing the technology of monolithic CMOS detectors for use as X-ray imaging spectrometers," This Proc., Paper 10397-2 (2017).

[16] Kenter, A., Kraft, R., Gauron, T., Murray, S.S., "Monolithic CMOS imaging x-ray spectrometers," Proc. SPIE 9154, 91540J (2014).

[17] Bandler, S.R., Adams, J.S., Chervenak, J.A., Datesman, A.M., Eckart, M.E., Finkbeiner, F.M., Kelley, R.L., Kilbourne, C.A., Betancourt-Martinez, G., Miniussi, A.R., Porter, F.S., Sadleir, J.E., Sakai, K., Smith, S.J., Stevenson, T.R., Wakeham, N.A., Wassell, E.J., Yoon, W., Becker, D., Bennett, D., Doriese, W.B., Fowler, J.W., Gard, J.D., Hilton, G.C., Mates, B., Morgan, K.M., Reintsema, C.D., Swetz, D., Ullom, J.N., Chaudhuri, S., Irwin, 
K.D., Lee, S.-J., Vikhlinin, A., "Development of x-ray microcalorimeter imaging spectrometers for the X-ray Surveyor mission concept," Proc. SPIE 99050Q (2016).

[18] Miles, D.M., McEntaffer, R.L., McCoy, J.A., DeRoo, C.T., Steiner, T., "Fabrication and diffraction efficiency of replicated, large-format X-ray reflection gratings," Opt. Letters, in prep (2017).

[19] DeRoo, C. T. McEntaffer, M. L, Donovan, B. McCoy, J., Miles, D., Tutt, J. Burwitz, V., Hartner, G., La Caria, MM, Pelliciari, C., Hartl, S. "X-ray Reflection Gratings Operated in an Echelle Mount," Light: Science \& Applications, in prep (2017).

[20] Heilmann, R. K., Bruccoleri, A.R., Song, J., Kolodziejczak, J., Gaskin, J.A., O’Dell, S.L., Cheimetz, P., Hertz, E., Smith, R.K., Burwitz, V., Hartner, G., La Caria, M.-M., Schattenburg, M.L., "Critical-angle transmission grating technology development for high resolving power soft x-ray spectrometers on Arcus and Lynx," This Proc. 10399 (2017).

[21] Heilmann, R. K., Bruccoleri, A.R., Kolodziejczak, J., Gaskin, J.A., O’Dell, S.L., Bhatia, R., Schattenburg, M.L., "Critical-angle x-ray transmission grating spectrometer with extended bandpass and resolving power > 10,000," Proc. SPIE 9905, 99051X (2016). 